\title{
Geometric-arithmetic Index and Zagreb Indices of Certain Special Molecular Graphs \\ Wenfei Xi ${ }^{1}$, Wei Gao ${ }^{2}$ \\ ${ }^{1}$ School of Tourism and Geographic Sciences, Yunnan Normal University Kunming 650500, China \\ ${ }^{2}$ School of Information Science and Technology, Yunnan Normal University \\ Kunming 650500, China
}

Abstract: In this paper, we determine the Geometric-arithmetic indexand Zagreb indicesof fan molecular graph, wheel molecular graph, gear fan molecular graph, gear wheel molecular graph, and their $r$-corona molecular graphs.

Keywords: Chemical graph theory, Geometric-arithmetic index, Zagreb index, Fan molecular graph, Wheel molecular graph, Gear fan moleculargraph, Gear wheel moleculargraph, $r$-corona moleculargraph

\section{$\underline{\text { Council for Innovative Research }}$}

Peer Review Research Publishing System

Journal: Journal of Advances in Chemistry

Vol. 10, No. 2

editorjaconline@gmail.com

www.cirjac.com 


\section{INTRODUCTION}

Wiener index,edge Wiener index, Hyper-wiener index, Geometric-arithmetic index and Zagreb indices are introduced to reflect certain structural features of organic molecules. Several papers contributed to determine the distance-based index of special molecular graphs (See Yan et al., [1] and [2], Gao and Shi [3] for more detail). Let $P_{n}$ and $C_{n}$ be path and cycle with $n$ vertices. The molecular graph $F_{n}=\{v\} \vee P_{n}$ is called a fan molecular graph and the molecular graph $W_{n}=\{v\} \vee C_{n}$ is called a wheel molecular graph. Molecular graph $I_{r}(G)$ is called $r$ - crown molecular graph of $G$ which splicing $r$ hang edges for every vertex in $G$. By adding one vertex in every two adjacent vertices of the fan path $P_{n}$ of fan molecular graph $F_{n}$, the resulting molecular graph is a subdivision molecular graph called gear fan molecular graph, denote as $\tilde{F}_{n}$. By adding one vertex in every two adjacent vertices of the wheel cycle $C_{n}$ of wheel molecular graph $W_{n}$, The resulting molecular graph is a subdivision molecular graph, called gear wheel molecular graph, denoted as $\tilde{W}_{n}$.

By considering the degrees of vertices in G, Vukicevic and Furtula [4]developed the Geometric-arithmetic index, shortly GA index, which is defined by

$$
G A(G)=\sum_{u v \in E(G)} \frac{2 \sqrt{d(u) d(v)}}{d(u)+d(v)},
$$

where $d(u)$ and $d(v)$ are the degrees of $u$ and $v$, respectively.

The (first and second) Zagreb indices have been introduced by Gutman and Trinajstic[5] as the form

$$
M_{1}(G)=\sum_{v \in V(G)}(d(v))^{2}
$$

and

$$
M_{2}(G)=\sum_{u v \in E(G)} d(u) d(v)
$$

On the other hand, for a moleculargraph $G$, the modified second Zagreb index $M_{2}^{*}(G)$ is defined as

$$
M_{2}^{*}(G)=\sum_{u v \in E(G)} \frac{1}{d(u) d(v)}
$$

Several papers contributed on determining the Zagreb indices of special molecular graphs can refer to [6-10].

In this paper, we present the Geometric-arithmetic index of $I_{r}\left(F_{n}\right), I_{r}\left(W_{n}\right), I_{r}\left(\tilde{F}_{n}\right)$ and $I_{r}\left(\tilde{W}_{n}\right)$. Also, the Zagreb indices of $I_{r}\left(F_{n}\right), I_{r}\left(W_{n}\right), I_{r}\left(\tilde{F}_{n}\right)$ and $I_{r}\left(\tilde{W}_{n}\right)$ are derived.

\section{GEOMETRIC-ARITHMETICINDEX}

Theorem 1. $G A\left(I_{r}\left(F_{n}\right)\right)=\frac{2 r \sqrt{n+r}}{n+r+1}+\frac{4 \sqrt{(n+r)(2+r)}}{n+2 r+2}+\frac{2(n-2) \sqrt{(n+r)(3+r)}}{n+2 r+3}$

$+\frac{4 \sqrt{(2+r)(3+r)}}{2 r+5}+\frac{(n-3) \sqrt{(3+r)(3+r)}}{r+3}+\frac{4 r \sqrt{2+r}}{r+3}+\frac{2(n-2) r \sqrt{3+r}}{r+4}$.

Proof. Let $P_{n}=v_{1} v_{2} \ldots v_{n}$ and the $r$ hanging vertices of $v_{\text {be }} v_{i}^{1}, v_{i}^{2}, \ldots, v_{i}^{r}(1 \leq i \leq n)$. Let $v$ be a vertex in $F_{n}$ beside $P_{n}$, and 
the $r$ hanging vertices of $v$ be $v^{1}, v^{2}, \ldots, v^{r}$. By the definition of Geometric-arithmetic index, we have

$$
\begin{aligned}
& G A\left(I_{r}\left(F_{n}\right)\right)=\sum_{i=1}^{r} \frac{2 \sqrt{d(v) d\left(v^{i}\right)}}{d(v)+d\left(v^{i}\right)}+\sum_{i=1}^{n} \frac{2 \sqrt{d(v) d\left(v_{i}\right)}}{d(v)+d\left(v_{i}\right)}+\sum_{i=1}^{n-1} \frac{2 \sqrt{d\left(v_{i}\right) d\left(v_{i+1}\right)}}{d\left(v_{i}\right)+d\left(v_{i+1}\right)}+\sum_{i=1}^{n} \sum_{j=1}^{r} \frac{2 \sqrt{d\left(v_{i}\right) d\left(v_{i}^{j}\right)}}{d\left(v_{i}\right)+d\left(v_{i}^{j}\right)} \\
& =\frac{2 r \sqrt{n+r}}{n+r+1}+\left(\frac{4 \sqrt{(n+r)(2+r)}}{n+2 r+2}+\frac{2(n-2) \sqrt{(n+r)(3+r)}}{n+2 r+3}\right) \\
& +\left(\frac{4 \sqrt{(2+r)(3+r)}}{2 r+5}+\frac{(n-3) \sqrt{(3+r)(3+r)}}{r+3}\right)+\left(\frac{4 r \sqrt{2+r}}{r+3}+\frac{2(n-2) r \sqrt{3+r}}{r+4}\right) .
\end{aligned}
$$

Corollary 1. $G A\left(F_{n}\right)=\frac{4 \sqrt{2 n}}{n+2}+\frac{2(n-2) \sqrt{3 n}}{n+3}+\frac{4 \sqrt{6}}{5}+n-3$.

Theorem 2. $G A\left(I_{r}\left(W_{n}\right)\right)=\frac{2 r \sqrt{n+r}}{n+r+1}+\frac{2 n \sqrt{(n+r)(3+r)}}{n+2 r+3}+\frac{n \sqrt{(3+r)(3+r)}}{r+3}+\frac{2 n r \sqrt{3+r}}{r+4}$.

Proof. Let $C_{n}=v_{1} v_{2} \ldots v_{n}$ and $v_{i}^{1}, v_{i}^{2}, \ldots, v_{i}^{r}$ be the $r$ hanging vertices of $v_{i}(1 \leq i \leq n)$. Let $v$ be a vertex in $W_{n}$ beside $C_{n}$, and $v^{1}, v^{2}, \ldots, v^{r}$ be the $r$ hanging vertices of $v$. By the definition of Geometric-arithmetic index, we have

$$
\begin{aligned}
& G A\left(I_{r}\left(W_{n}\right)\right)=\sum_{i=1}^{r} \frac{2 \sqrt{d(v) d\left(v^{i}\right)}}{d(v)+d\left(v^{i}\right)}+\sum_{i=1}^{n} \frac{2 \sqrt{d(v) d\left(v_{i}\right)}}{d(v)+d\left(v_{i}\right)}+\sum_{i=1}^{n} \frac{2 \sqrt{d\left(v_{i}\right) d\left(v_{i+1}\right)}}{d\left(v_{i}\right)+d\left(v_{i+1}\right)}+\sum_{i=1}^{n} \sum_{j=1}^{r} \frac{2 \sqrt{d\left(v_{i}\right) d\left(v_{i}^{j}\right)}}{d\left(v_{i}\right)+d\left(v_{i}^{j}\right)} \\
& =\frac{2 r \sqrt{n+r}}{n+r+1}+\frac{2 n \sqrt{(n+r)(3+r)}}{n+2 r+3}+\frac{n \sqrt{(3+r)(3+r)}}{r+3}+\frac{2 n r \sqrt{3+r}}{r+4} .
\end{aligned}
$$

Corollary 2. $G A\left(W_{n}\right)=\frac{2 n \sqrt{3 n}}{n+3}+n$

Theorem 3. $G A\left(I_{r}\left(\tilde{F}_{n}\right)\right)=\frac{2 r \sqrt{n+r}}{n+r+1}+\frac{4 \sqrt{(n+r)(2+r)}}{n+2 r+2}+\frac{2(n-2) \sqrt{(n+r)(3+r)}}{n+2 r+3}$

$+\frac{4 r \sqrt{2+r}}{r+3}+\frac{2(n-2) r \sqrt{3+r}}{r+4}+\frac{2 \sqrt{(2+r)(2+r)}}{r+2}+\frac{4(n-2) \sqrt{(3+r)(2+r)}}{2 r+5}+\frac{2(n-1) r \sqrt{2+r}}{r+3}$.

Proof.Let $P_{n}=v_{1} v_{2} \ldots v_{n}$ and $v_{i, i+1}$ be the adding vertex between $v_{i}$ and $v_{i+1}$. Let $v_{i}^{1}, v_{i}^{2}, \ldots, v_{i}^{r}$ be the $r$ hanging vertices of $v_{i}(1$ $\leq i \leq n)$. Let $v_{i, i+1}^{1}, v_{i, i+1}^{2}, \ldots, v_{i, i+1}^{r}$ be the $r$ hanging vertices of $v_{i, i+1}(1 \leq i \leq n-1)$. Let $v$ be a vertex in $F_{n}$ beside $P_{n}$, and the $r$ hanging vertices of $v$ be $v^{1}, v^{2}, \ldots, v^{r}$. By virtue of the definition of Geometric-arithmetic index, we get 
$G A\left(I_{r}\left(\tilde{F}_{n}\right)\right)=\sum_{i=1}^{r} \frac{2 \sqrt{d(v) d\left(v^{i}\right)}}{d(v)+d\left(v^{i}\right)}+\sum_{i=1}^{n} \frac{2 \sqrt{d(v) d\left(v_{i}\right)}}{d(v)+d\left(v_{i}\right)}+\sum_{i=1}^{n} \sum_{j=1}^{r} \frac{2 \sqrt{d\left(v_{i}\right) d\left(v_{i}^{j}\right)}}{d\left(v_{i}\right)+d\left(v_{i}^{j}\right)}+\sum_{i=1}^{n-1} \frac{2 \sqrt{d\left(v_{i}\right) d\left(v_{i, i+1}\right)}}{d\left(v_{i}\right)+d\left(v_{i, i+1}\right)}+$ $\sum_{i=1}^{n-1} \frac{2 \sqrt{d\left(v_{i, i+1}\right) d\left(v_{i+1}\right)}}{d\left(v_{i, i+1}\right)+d\left(v_{i+1}\right)}+\sum_{i=1}^{n-1} \sum_{j=1}^{r} \frac{2 \sqrt{d\left(v_{i, i+1}\right) d\left(v_{i, i+1}^{j}\right)}}{d\left(v_{i, i+1}\right)+d\left(v_{i, i+1}^{j}\right)}$

$=\frac{2 r \sqrt{n+r}}{n+r+1}+\left(\frac{4 \sqrt{(n+r)(2+r)}}{n+2 r+2}+\frac{2(n-2) \sqrt{(n+r)(3+r)}}{n+2 r+3}\right)$

$+\left(\frac{4 r \sqrt{2+r}}{r+3}+\frac{2(n-2) r \sqrt{3+r}}{r+4}\right)+\left(\frac{\sqrt{(2+r)(2+r)}}{r+2}+\frac{2(n-2) \sqrt{(3+r)(2+r)}}{2 r+5}\right)$

$+\left(\frac{\sqrt{(2+r)(2+r)}}{r+2}+\frac{2(n-2) \sqrt{(3+r)(2+r)}}{2 r+5}\right)+\frac{2(n-1) r \sqrt{2+r}}{r+3}$.

$\square$ Corollary3. $G A\left(\tilde{F}_{n}\right)=\frac{4 \sqrt{2 n}}{n+2}+\frac{2(n-2) \sqrt{3 n}}{n+3}+\frac{4(n-2) \sqrt{6}+10}{5}$

Theorem4. $G A\left(I_{r}\left(\tilde{W}_{n}\right)\right)=\frac{2 r \sqrt{n+r}}{n+r+1}+\frac{2 n \sqrt{(n+r)(3+r)}}{n+2 r+3}+\frac{2 n r \sqrt{3+r}}{r+4}+\frac{4 n \sqrt{(3+r)(2+r)}}{2 r+5}+\frac{2 n r \sqrt{2+r}}{r+3}$.

Proof. Let $C_{n}=v_{1} v_{2} \ldots v_{n}$ and $v$ be a vertex in $W_{n}$ beside $C_{n}, v_{i, i+1} \square$ be the adding vertex between $v_{i}$ and $v_{i+1}$. Let $v^{1}, v^{2}, \ldots$, $v^{r}$ be the $r$ hanging vertices of $v$ and $v_{i}^{1}, v_{i}^{2}, \ldots, v_{i}^{r}$ be the $r$ hanging vertices of $v_{i}(1 \leq i \leq n)$. Let $v_{n, n+1}=v_{1, n}$ and $v_{i, i+1}^{1}$, $v_{i, i+1}^{2}, \ldots, v_{i, i+1}^{r}$ be the $r$ hanging vertices of $v_{i, i+1}(1 \leq i \leq n)$. In view of the definition of Geometric-arithmetic index, we deduce

$G A\left(I_{r}\left(\tilde{W}_{n}\right)\right)=\sum_{i=1}^{r} \frac{2 \sqrt{d(v) d\left(v^{i}\right)}}{d(v)+d\left(v^{i}\right)}+\sum_{i=1}^{n} \frac{2 \sqrt{d(v) d\left(v_{i}\right)}}{d(v)+d\left(v_{i}\right)}+\sum_{i=1}^{n} \sum_{j=1}^{r} \frac{2 \sqrt{d\left(v_{i}\right) d\left(v_{i}^{j}\right)}}{d\left(v_{i}\right)+d\left(v_{i}^{j}\right)}+\sum_{i=1}^{n} \frac{2 \sqrt{d\left(v_{i}\right) d\left(v_{i, i+1}\right)}}{d\left(v_{i}\right)+d\left(v_{i, i+1}\right)}+$ $\sum_{i=1}^{n} \frac{2 \sqrt{d\left(v_{i, i+1}\right) d\left(v_{i+1}\right)}}{d\left(v_{i, i+1}\right)+d\left(v_{i+1}\right)}+\sum_{i=1}^{n} \sum_{j=1}^{r} \frac{2 \sqrt{d\left(v_{i, i+1}\right) d\left(v_{i, i+1}^{j}\right)}}{d\left(v_{i, i+1}\right)+d\left(v_{i, i+1}^{j}\right)}$

$=\frac{2 r \sqrt{n+r}}{n+r+1}+\frac{2 n \sqrt{(n+r)(3+r)}}{n+2 r+3}+\frac{2 n r \sqrt{3+r}}{r+4}+\frac{2 n \sqrt{(3+r)(2+r)}}{2 r+5}+\frac{2 n \sqrt{(3+r)(2+r)}}{2 r+5}+\frac{2 n r \sqrt{2+r}}{r+3}$

Corollary 4. $G A\left(\tilde{W}_{n}\right)=\frac{2 n \sqrt{3 n}}{n+3}+\frac{4 n \sqrt{6}}{5}$.

3. ZAGREB INDICES

Using the notations defined in above section, and combining with the definitions of Zagreb indices, we get the following computational formulas. 


$$
\begin{aligned}
& M_{1}\left(I_{r}\left(F_{n}\right)\right)=(d(v))^{2}+\sum_{i=1}^{r}\left(d\left(v^{i}\right)\right)^{2}+\sum_{i=1}^{n}\left(d\left(v_{i}\right)\right)^{2}+\sum_{i=1}^{n} \sum_{j=1}^{r}\left(d\left(v_{i}^{j}\right)\right)^{2} \\
& =(n+r)^{2}+r+2(2+r)^{2}+(n-2)(3+r)^{2}+n r \\
& =r^{2}(n+1)+r(9 n-3)+n^{2}+9 n-10 . \\
& M_{1}\left(F_{n}\right)=n^{2}+9 n-10 . \\
& M_{1}\left(I_{r}\left(W_{n}\right)\right)=(d(v))^{2}+\sum_{i=1}^{r}\left(d\left(v^{i}\right)\right)^{2}+\sum_{i=1}^{n}\left(d\left(v_{i}\right)\right)^{2}+\sum_{i=1}^{n} \sum_{j=1}^{r}\left(d\left(v_{i}^{j}\right)\right)^{2} \\
& =(n+r)^{2}+r+n(3+r)^{2}+n r \\
& =r^{2}(n+1)+r(9 n+1)+n^{2}+9 n \text {. } \\
& M_{1}\left(W_{n}\right)=n^{2}+9 n \\
& M_{1}\left(I_{r}\left(\tilde{F}_{n}\right)\right)=(d(v))^{2}+\sum_{i=1}^{r}\left(d\left(v^{i}\right)\right)^{2}+\sum_{i=1}^{n}\left(d\left(v_{i}\right)\right)^{2}+\sum_{i=1}^{n} \sum_{j=1}^{r}\left(d\left(v_{i}^{j}\right)\right)^{2}+\sum_{i=1}^{n-1}\left(d\left(v_{i, i+1}\right)\right)^{2} \\
& +\sum_{i=1}^{n-1} \sum_{j=1}^{r}\left(d\left(v_{i, i+1}^{j}\right)\right)^{2} \\
& =(n+r)^{2}+r+2(2+r)^{2}+(n-2)(3+r)^{2}+n r+(n-1)(2+r)^{2}+r(n-1) \\
& =2 n r^{2}+r(14 n-8)+n^{2}+13 n-14 \text {. } \\
& M_{1}\left(\tilde{F}_{n}\right)=n^{2}+13 n-14 \\
& M_{1}\left(I_{r}\left(\tilde{W}_{n}\right)\right)=(d(v))^{2}+\sum_{i=1}^{r}\left(d\left(v^{i}\right)\right)^{2}+\sum_{i=1}^{n}\left(d\left(v_{i}\right)\right)^{2}+\sum_{i=1}^{n} \sum_{j=1}^{r}\left(d\left(v_{i}^{j}\right)\right)^{2}+\sum_{i=1}^{n}\left(d\left(v_{i, i+1}\right)\right)^{2} \\
& +\sum_{i=1}^{n} \sum_{j=1}^{r}\left(d\left(v_{i, i+1}^{j}\right)\right)^{2} \\
& =(n+r)^{2}+r+n(3+r)^{2}+n r+n(2+r)^{2}+n r \\
& =r^{2}(2 n+1)+r(14 n+1)+n^{2}+13 n \text {. }
\end{aligned}
$$

$M_{1}\left(\tilde{W}_{n}\right)=n^{2}+13 n$

$M_{2}\left(I_{r}\left(F_{n}\right)\right)=\sum_{i=1}^{r} d(v) d\left(v^{i}\right)+\sum_{i=1}^{n} d(v) d\left(v_{i}\right)+\sum_{i=1}^{n-1} d\left(v_{i}\right) d\left(v_{i+1}\right)+\sum_{i=1}^{n} \sum_{j=1}^{r} d\left(v_{i}\right) d\left(v_{i}^{j}\right)$ 


$$
\begin{aligned}
& =r(n+r)+(2(n+r)(2+r)+(n-2)(n+r)(3+r))+(2(2+r)(3+r)+(n-3)(3+r)(3+r)) \\
& +(2 r(2+r)+(n-2) r(3+r)) \\
& =3 r^{2} n+r\left(n^{2}+13 n-12\right)+3 n^{2}+7 n-15 \text {. } \\
& M_{2}\left(F_{n}\right)=3 n^{2}+7 n-15 \text {. } \\
& M_{2}\left(I_{r}\left(W_{n}\right)\right)=\sum_{i=1}^{r} d(v) d\left(v^{i}\right)+\sum_{i=1}^{n} d(v) d\left(v_{i}\right)+\sum_{i=1}^{n} d\left(v_{i}\right) d\left(v_{i+1}\right)+\sum_{i=1}^{n} \sum_{j=1}^{r} d\left(v_{i}\right) d\left(v_{i}^{j}\right) \\
& =r(n+r)+n(n+r)(3+r)+n(3+r)(3+r)+n r(3+r) . \\
& =r^{2}(3 n+1)+r\left(n^{2}+13 n\right)+3 n^{2}+9 n \\
& M_{2}\left(W_{n}\right)=3 n^{2}+9 n \\
& M_{2}\left(I_{r}\left(\tilde{F}_{n}\right)\right)=\sum_{i=1}^{r} d(v) d\left(v^{i}\right)+\sum_{i=1}^{n} d(v) d\left(v_{i}\right)+\sum_{i=1}^{n} \sum_{j=1}^{r} d\left(v_{i}\right) d\left(v_{i}^{j}\right)+\sum_{i=1}^{n-1} d\left(v_{i}\right) d\left(v_{i, i+1}\right)+\sum_{i=1}^{n-1} d\left(v_{i, i+1}\right) d\left(v_{i+1}\right)+ \\
& \sum_{i=1}^{n-1} \sum_{j=1}^{r} d\left(v_{i, i+1}\right) d\left(v_{i, i+1}^{j}\right) \\
& =r(n+r)+(2(n+r)(2+r)+(n-2)(n+r)(3+r))+(2 r(2+r)+(n-2) r(3+r))+ \\
& ((2+r)(2+r)+(n-2)(3+r)(2+r))+((2+r)(2+r)+(n-2)(3+r)(2+r)) \\
& +(n-1) r(2+r) \\
& =r^{2}(5 n-3)+r\left(n^{2}+19 n-18\right)+3 n^{2}+10 n-16 . \\
& M_{2}\left(\tilde{F}_{n}\right)=3 n^{2}+10 n-16 \\
& M_{2}\left(I_{r}\left(\tilde{W}_{n}\right)\right)=\sum_{i=1}^{r} d(v) d\left(v^{i}\right)+\sum_{i=1}^{n} d(v) d\left(v_{i}\right)+\sum_{i=1}^{n} \sum_{j=1}^{r} d\left(v_{i}\right) d\left(v_{i}^{j}\right)+\sum_{i=1}^{n} d\left(v_{i}\right) d\left(v_{i, i+1}\right)+\sum_{i=1}^{n} d\left(v_{i, i+1}\right) d\left(v_{i+1}\right)+ \\
& \sum_{i=1}^{n} \sum_{j=1}^{r} d\left(v_{i, i+1}\right) d\left(v_{i, i+1}^{j}\right) \\
& =r(n+r)+n(n+r)(3+r)+n r(3+r)+n(3+r)(2+r)+n(3+r)(2+r)+n r(2+r) \\
& =r^{2}(5 n+1)+r\left(n^{2}+19 n\right)+3 n^{2}+12 n \\
& M_{2}\left(\tilde{W}_{n}\right)=3 n^{2}+12 n .
\end{aligned}
$$




$$
\begin{aligned}
& M_{2}^{*}\left(I_{r}\left(F_{n}\right)\right)=\sum_{i=1}^{r}\left(d(v) d\left(v^{i}\right)\right)^{-1}+\sum_{i=1}^{n}\left(d(v) d\left(v_{i}\right)\right)^{-1}+\sum_{i=1}^{n-1}\left(d\left(v_{i}\right) d\left(v_{i+1}\right)\right)^{-1}+\sum_{i=1}^{n} \sum_{j=1}^{r}\left(d\left(v_{i}\right) d\left(v_{i}^{j}\right)\right)^{-1} \\
& =\frac{r}{n+r}+\frac{2}{(n+r)(2+r)}+\frac{n-2}{(n+r)(3+r)}+\frac{2}{(2+r)(3+r)}+\frac{n-3}{(3+r)(3+r)} \\
& +\frac{2 r}{2+r}+\frac{(n-2) r}{3+r} \text {. } \\
& M_{2}^{*}\left(F_{n}\right)=\frac{1}{n}+\frac{n-2}{3 n}+\frac{1}{3}+\frac{n-3}{9} . \\
& M_{2}^{*}\left(I_{r}\left(W_{n}\right)\right)=\sum_{i=1}^{r}\left(d(v) d\left(v^{i}\right)\right)^{-1}+\sum_{i=1}^{n}\left(d(v) d\left(v_{i}\right)\right)^{-1}+\sum_{i=1}^{n}\left(d\left(v_{i}\right) d\left(v_{i+1}\right)\right)^{-1}+\sum_{i=1}^{n} \sum_{j=1}^{r}\left(d\left(v_{i}\right) d\left(v_{i}^{j}\right)\right)^{-1} \\
& =\frac{r}{n+r}+\frac{n}{(n+r)(3+r)}+\frac{n}{(3+r)(3+r)}+\frac{n r}{3+r} . \\
& M_{2}^{*}\left(W_{n}\right)=\frac{1}{3}+\frac{n}{9} \\
& M_{2}^{*}\left(I_{r}\left(\tilde{F}_{n}\right)\right)=\sum_{i=1}^{r}\left(d(v) d\left(v^{i}\right)\right)^{-1}+\sum_{i=1}^{n}\left(d(v) d\left(v_{i}\right)\right)^{-1}+\sum_{i=1}^{n} \sum_{j=1}^{r}\left(d\left(v_{i}\right) d\left(v_{i}^{j}\right)\right)^{-1}+\sum_{i=1}^{n-1}\left(d\left(v_{i}\right) d\left(v_{i, i+1}\right)\right)^{-1}+ \\
& \sum_{i=1}^{n-1}\left(d\left(v_{i, i+1}\right) d\left(v_{i+1}\right)\right)^{-1}+\sum_{i=1}^{n-1} \sum_{j=1}^{r}\left(d\left(v_{i, i+1}\right) d\left(v_{i, i+1}^{j}\right)\right)^{-1} \\
& =\frac{r}{n+r}+\frac{2}{(n+r)(2+r)}+\frac{n-2}{(n+r)(3+r)}+\frac{2 r}{2+r}+\frac{(n-2) r}{3+r}+\frac{2}{(2+r)(2+r)}+\frac{2(n-2)}{(3+r)(2+r)}+\frac{(n-1) r}{2+r} . \\
& M_{2}^{*}\left(\tilde{F}_{n}\right)=\frac{1}{n}+\frac{n-2}{3 n}+\frac{1}{2}+\frac{n-2}{3} . \\
& M_{2}^{*}\left(I_{r}\left(\tilde{W}_{n}\right)\right)=\sum_{i=1}^{r}\left(d(v) d\left(v^{i}\right)\right)^{-1}+\sum_{i=1}^{n}\left(d(v) d\left(v_{i}\right)\right)^{-1}+\sum_{i=1}^{n} \sum_{j=1}^{r}\left(d\left(v_{i}\right) d\left(v_{i}^{j}\right)\right)^{-1}+\sum_{i=1}^{n}\left(d\left(v_{i}\right) d\left(v_{i, i+1}\right)\right)^{-1}+ \\
& \sum_{i=1}^{n}\left(d\left(v_{i, i+1}\right) d\left(v_{i+1}\right)\right)^{-1}+\sum_{i=1}^{n} \sum_{j=1}^{r}\left(d\left(v_{i, i+1}\right) d\left(v_{i, i+1}^{j}\right)\right)^{-1} \\
& =\frac{r}{n+r}+\frac{n}{(n+r)(3+r)}+\frac{n r}{3+r}+\frac{2 n}{(3+r)(2+r)}+\frac{n r}{2+r} . \\
& M_{2}^{*}\left(\tilde{W}_{n}\right)=\frac{n+1}{3} .
\end{aligned}
$$




\section{CONCLUSION}

In this paper, we determine the Geometric-arithmetic index and Zagreb indices of fan molecular graph, wheel molecular graph, gear fan molecular graph, gear wheel molecular graph, and their $r$-corona molecular graphs.

\section{Acknowledgements}

First, we thank the reviewers for their constructive comments in improving the quality of this paper. This work was supported in part by the National Natural Science Foundation of China (61262071), and the Key Science and Technology Research Project of Education Ministry (210210). We also would like to thank the anonymous referees for providing us with constructive comments and suggestions.

\section{REFERENCES}

[1] L. Yan, Y. Li, W. Gao, J. Li, On the external hyper-wiener index of graphs, Journal of Chemical and Pharmaceutical Research, 2014, 6(3):477-481.

[2] L. Yan, Y. Li, W. Gao, J. Li, PI index for some special graphs, Journal of Chemical and Pharmaceutical Research, 2013, 5(11):260-264.

[3] W. Gao, L. Shi, Wiener index of gear fan graph and gear wheel graph, Asian Journal of Chemistry, 2014, 26(11): 3397-3400.

[4] D. Vukicevic, B. Furtula, Topological index based on the ratios of geometrical and arithmetical means of end-vertexdegrees of edges, J. Math. Chem.,2009, 4: 1369-1376.

[5] I. Gutman, N.Trinajstic, Graph theory and molecular orbitals. Total $\frac{1}{4}$-electron energy of alternate hydrocarbons.Chem. Phys. Lett., 1972, 17: 535-538.

[6] K. Das, I.Gutman, B. Zhou, New upper bounds on Zagreb indices, J. Math. Chem., 2009, 46, 514-521.

[7] P. Ranjini, V.Lokesha, The Smarandache-Zagreb indices on the three graph operators, Int. J. Math.Combin.,2010, 3: 1-10.

[8] P. Ranjini, V.Lokesha, I.Cangul, On the Zagreb indices of the line graphs of the subdivision graphs. Appl. Math.Comput., 2011, 218(3): 699-702.

[9] P. Ranjini, V.Lokesha, M.Rajan, On Zagreb indices of the subdivision graphs, Int. J. Math. Sci. Eng. Appl.,2010, 4: 221-228.

[10] K. Das, Atom-bond connectivity index of graphs, Discrete Appl. Math., 2010, 158: 1181-1188. 